\title{
Characterization of Yeast Biofilm by Cryo-SEM and FIB-SEM
}

\author{
Kamila Dobranska $^{1,2}$, Jana Nebesarova ${ }^{3}$, Filip Ruzicka ${ }^{4,5}$, Jiri Dluhos ${ }^{6}$, Vladislav Krzyzanek ${ }^{1}$
}

1. Institute of Scientific Instrument ASCR, Department of Electron Microscopy, Brno, Czech Republic

2. Brno University of Technology, Institute of Physical Engineering, Brno, Czech Republic

3. Biology center ASCR, Institute of Parasitology, Ceske Budejovice, Czech Republic

4. Masaryk University, Faculty of Medicine, Brno, Czech Republic

5. FNSUA, Department of Microbiology, Brno, Czech Republic

6. TESCAN, Brno, Czech Republic

Microscopic organisms include all of the prokaryotes, namely the bacteria; and various forms of eukaryote such as yeast that have been studied. Besides the planktonic way of living, microbes are able to adhere to surfaces or interfaces and to form organized communities, a so-called biofilm, which are embedded in a matrix of extracellular polymeric substances that they produce. These communities are beneficial to humans as microflora colonizing surfaces of the human body. On the other hand the growth of biofilm may bring many problems. In medicine the biofilm formation allows microorganisms to colonize the surface of implants and it also protects the microbial cells from attacks by the immunity system as well as from the effect of antibiotics. Therefore, the biofilm is considered to be important virulence factor in these microorganisms. The characteristic features of the biofilm infections, especially high resistance to antifungal agents, complicate therapy [1].

The structure of the biofilm layer and proportion and composition of extracellular matrix differs by type of organism and environmental conditions, where a bacterial/yeast culture is evolving and growing. Understanding of the biofilm structure can contribute to understanding the biofilm formation and basic biochemical mechanisms underlying this process. It may help to develop more efficient treatment strategy for biofilm infection.

Yeasts like Candida parapsilosis as well as Candida albicans has been recently recognized as an important cause of serious biofilm infections associated with implanted medical devices. The multilayered biofilms formed by these microorganisms were observed by cryo-scanning electron microscope (cryo-SEM) with using freeze-fracturing technique [2] and by focused ion beam scanning electron microscopy (FIB-SEM). Both imaging methods are compared.

The freeze-fracture technique consists of physically breaking apart (fracturing) a rapidly frozen biological sample; structural detail exposed by the fracture plane may be then visualized by metal deposition. Our samples were fractured after rapid freezing into liquid nitrogen or into liquid propane, then followed short sublimation of ice contamination (caused e.g. by air humidity) by freeze-drying at Alto 2500 chamber (Gatan), sputtered by $5 \mathrm{~nm}$ Pt-Pd and finally imaged at low temperature in the field emission SEM JSM 7401F (JEOL) (Figure 1).

Experiments with FIB-SEM have been done using the microscope LYRA FIB-FESEM (Tescan). Sample preparation included high pressure freezing (Leica EM PACT2) that is considered as the most effective fixation method of full hydrated materials, because it is possible to freeze layer up to $200 \mu \mathrm{m}$ thick without ice crystallization, then followed automatic freeze substitution (Leica EMAFS) and embedding into Epon resin (Figure 2). 
Cryo-SEM is an excellent technique for imaging liquid and semi-liquid materials in a very close state to the native structure with quite high resolution. As well as FIB-SEM is satisfactory method to observe and study biological materials [3]. Therefore, both techniques were chosen and the comparison of result brings useful information about yeast biofilm.

[1] RM Donlan and JW Costerton, Clin. Microbiol. Rev. 15 (2002), p. 167.

[2] H Moor and K Mühlethaler, Rockefeller University Press 17 (1963), p. 609.

[3] M Milani and D Drobne, Scanning 28 (2006), p. 148.

[4] The authors acknowledge the support by the grant CZ.1.07/2.3.00/20.0103 (EC and MEYS CR), TE01020118 (TACR) and by GACR (project P205/11/1687).
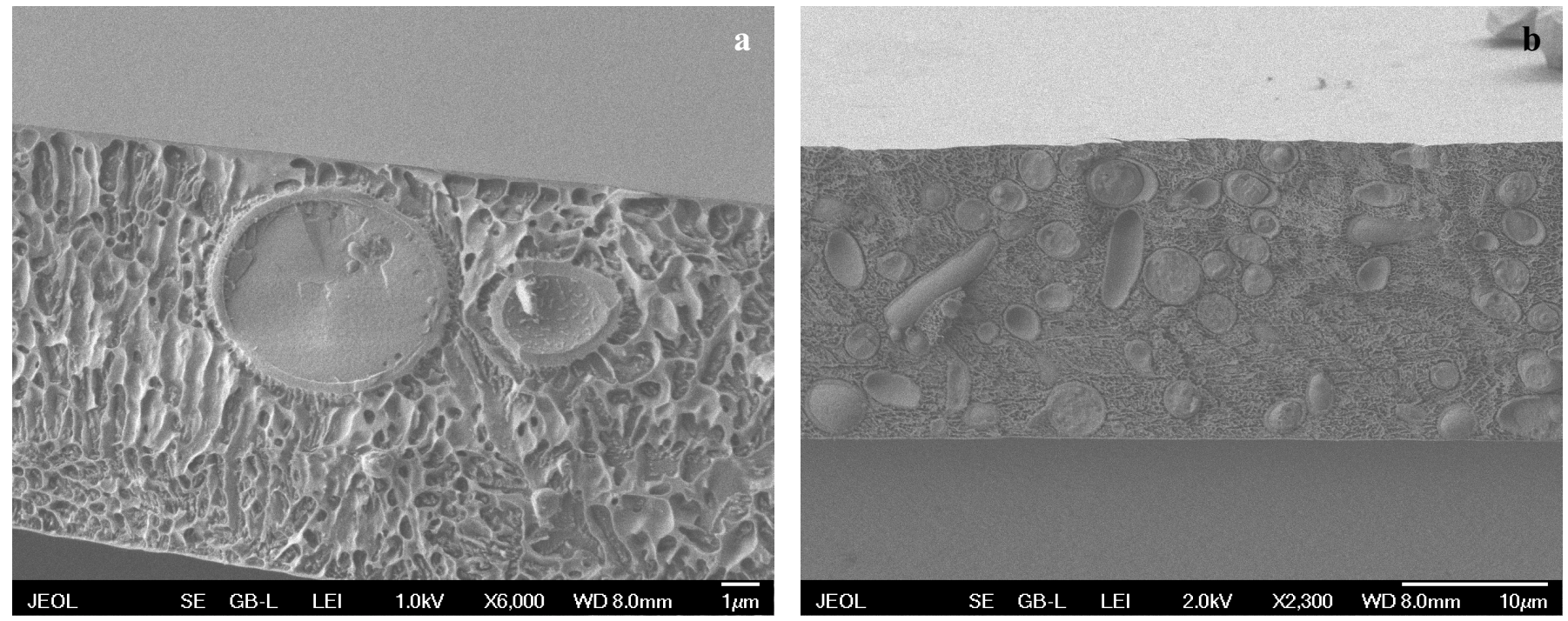

Figure 1. (a) Candida albicans grown on cover glass frozen in liquid propane; (b) Candida parapsilosis grown on cover glass frozen in liquid nitrogen.
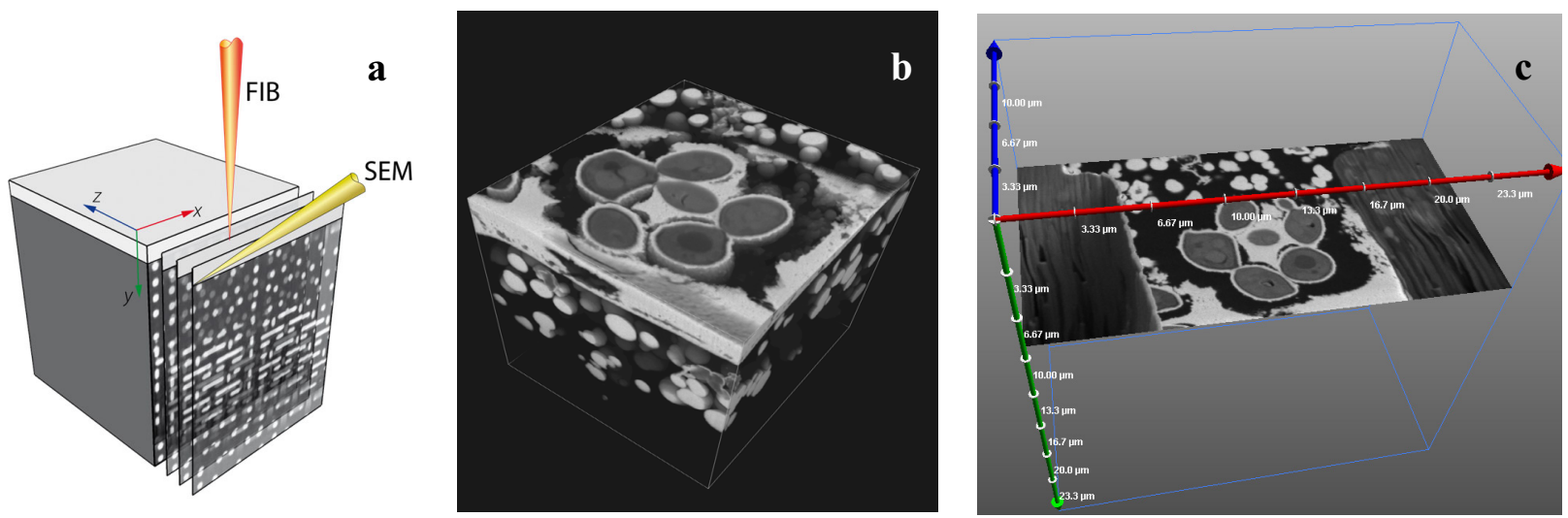

Figure 2. (a) Principle of FIB-SEM tomography; (b, c) Candida albicans visualizations of FIB-SEM tomography reconstructions. 